\title{
On the validity of eikonal equation for surface-wave phase-velocity tomography
}

\author{
M. Lehujeur ${ }^{1}$, S. Chevrot ${ }^{1}$ \\ ${ }^{1}$ GET, UMR 5563, Observatoire Midi Pyrénées, Université Paul Sabatier, CNRS, IRD, Toulouse, France
}

\begin{abstract}
SUMMARY
The phase velocity of surface waves can be directly determined from the amplitude and phase of the regional wavefield using the Helmholtz equation. However, the Helmholtz equation involves estimating the Laplacian of the amplitude field, a challenging operation to perform on noisy and sparsely sampled seismic data. For this reason, the amplitude information is often discarded. In that case, phase velocity maps are reconstructed with the eikonal equation, which relates the local phase slowness to the gradient of the phase. Here, we derive analytical expression of the errors arising from neglecting the amplitude of the wavefield in eikonal tomography. In general, these errors are quite strong but they vary sinusoidally with the wave propagation direction. Consequently, if the azimuthal coverage is good, they will average out, and unbiased phase velocity maps can be obtained with eikonal tomography. We numerically validate these results with a synthetic tomography experiment.
\end{abstract}

Key words: Seismology, Tomography, Surface waves and free oscillations

\section{INTRODUCTION}

The Helmholtz equation relates the travel time and amplitude fields to the local phase velocity of surface waves, thereby providing a direct means to map lateral variations of phase velocities beneath dense regional arrays (Friederich et al. 1994; Friederich \& Wielandt 1995). The main advantage of Helmholtz tomography, apart from its implementation simplicity, is that it does not involve resolving 


\section{Lehujeur and Chevrot}

an inverse problem, thus also avoiding degrading the resolution by introducing arbitrary damping and smoothing regularization constraints into the inversion. Accompanying the booming of dense regional seismic deployments, Helmholtz tomography rapidly developed and gained in popularity. Perhaps its most remarkable achievements came from the exploitation of the USArray Transportable Array (TA) data to image the shear velocity structure of the crust and uppermost mantle beneath USA with unprecedented resolution (Pollitz 2008; Lin et al. 2009; Lin \& Ritzwoller 2011; Jin \& Gaherty 2015; Shen \& Ritzwoller 2016).

Helmholtz tomography involves estimating the Laplacian, i.e. second order spatial derivatives, of the amplitude field from its samples at the position of seismological stations. This is an ill-posed problem because differentiation is very sensitive to noise (Scales \& Tenorio 2001; Roy 2015). In practice, resulting amplitude corrections are often found to be small compared to the uncertainty on phase velocity maps (e.g., Feng \& Ritzwoller 2019) and are often ignored. Ignoring the amplitude information in the Helmholtz equation leads to the eikonal equation, which simply relates the gradient of the phase to the local slowness (Wielandt 1993; Lin et al. 2009). Nevertheless, even though eikonal tomography leads to phase velocity maps with strong artefacts (e.g., Wielandt 1993; Friederich et al. 1994), Bodin $\&$ Maupin (2008) have shown that these artefacts can be strongly mitigated if the azimuthal coverage is good.

In this study, we characterize the errors introduced by neglecting the contribution of amplitude in the reconstruction of the velocity model, i.e. of using the eikonal equation instead of the complete Helmholtz equation. We discuss the limits of validity of eikonal tomography and suggest simple procedures to improve the quality and robustness of resulting phase velocity models. We then perform a synthetic eikonal tomography experiment to numerically validate our results.

\section{THEORETICAL CONSIDERATIONS}

\subsection{The Helmholtz equation}

In a smoothly varying Earth model, Love and Rayleigh wave displacements can be represented in terms of a 2-D potential $\chi$ that satisfy the Helmholtz equation (Tanimoto 1990; Tromp \& Dahlen 1993)

$$
\Delta \chi(\mathbf{r})+\frac{\omega^{2}}{c^{2}} \chi(\mathbf{r})=0
$$

where $\Delta$ is the Laplacian operator, $\mathbf{r}$ the position vector, $\omega$ the angular frequency, and $c$ the local Love or Rayleigh phase velocity. 
A simple high-frequency solution of (1) is given by

$$
\chi(\mathbf{r})=A(\mathbf{r}) e^{i \omega T(\mathbf{r})}
$$

where $A(\mathbf{r})$ and $T(\mathbf{r})$ are respectively the amplitude and phase of potential $\chi$ at location $\mathbf{r}$. Equation (2) is valid if $A(\mathbf{r})$ and $T(\mathbf{r})$ are both smooth functions of spatial coordinates. In that case, the Helmholtz equation (1) can be rewritten in the form (e.g., Červený 2001)

$$
\Delta A-\omega^{2} A\|\nabla T\|^{2}+\frac{\omega^{2}}{c^{2}} A+i \omega(2 \nabla A \cdot \nabla T+A \Delta T)=0,
$$

where for the sake of conciseness we omitted the spatial dependences of $A$ and $T$. Isolating the imaginary part of (3) gives the transport equation

$$
2 \nabla A \cdot \nabla T+A \Delta T=0
$$

whereas the real part leads to (Wielandt 1993)

$$
\frac{1}{c^{2}}=\|\nabla T\|^{2}-\frac{\Delta A}{\omega^{2} A},
$$

which is often referred to as the Helmholtz equation in the literature (e.g., Lin et al. 2012; Jin \& Gaherty 2015). This equation provides a direct relationship between the local phase velocity and the phase and amplitude of a surface-wave front, two quantities that can be easily determined from seismic records.

\subsection{The eikonal equation}

The main limitation of Helmholtz tomography comes from the second term on the right side of (5). Its estimation involves computing the Laplacian of the amplitude field, i.e. computing its second derivatives, a challenging task because of noise measurement and irregular sparse station sampling. Ignoring the amplitude term leads to the eikonal equation (Lin et al. 2009):

$$
\frac{1}{c^{2}}=\|\nabla T\|^{2}
$$

In that case, the gradient of the traveltime field is directly related to the local phase slowness.

The validity of the eikonal equation is often justified by the $\omega^{-2}$ dependence of the amplitude term, thus considered as a finite-frequency correction that can be neglected at high frequency (e.g., Lin et al. 2009). Numerical experiments have demonstrated that the amplitude field is controlled by the interference pattern between the incoming and scattered wavefields (Friederich et al. 2000; Bodin $\&$ Maupin 2008). These scattered waves, as well as the complexities of the incoming wavefield, can strongly bias phase velocity estimates relying purely on phase information (Wielandt 1993; Friederich \& Dalkolmo 1995; Friederich \& Wielandt 1995; Pollitz 2008; Pollitz \& Snoke 2010). Observations 


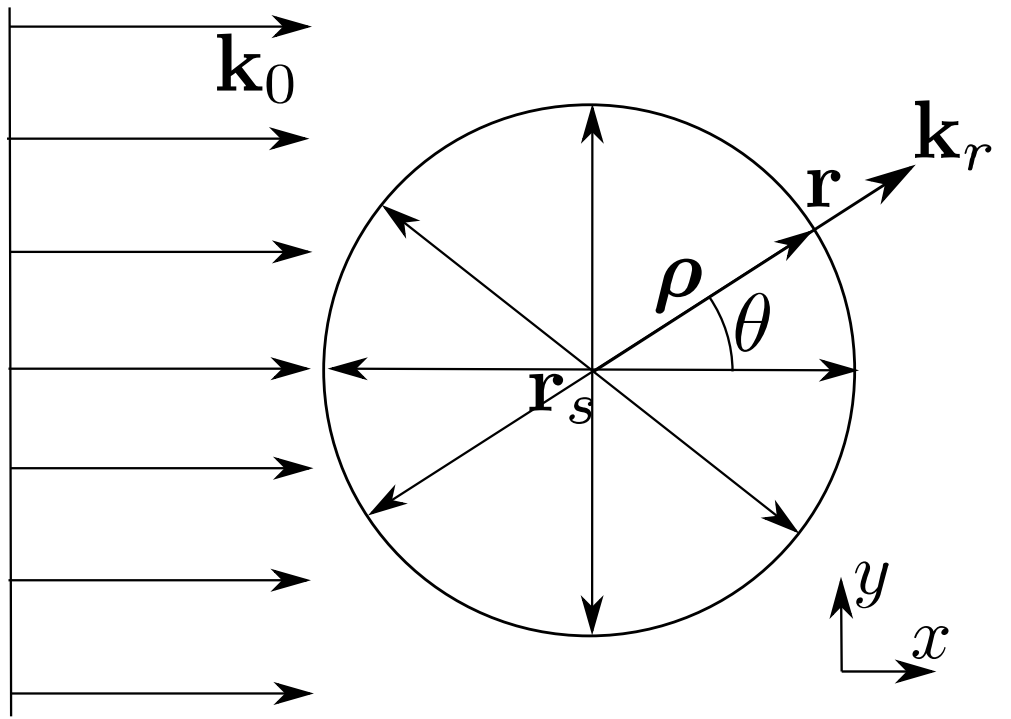

Figure 1. Eikonal tomography in the case of a point scatterer located at $\mathbf{r}_{s}$ inside a homogeneous background medium. We consider an incoming plane wave with wave vector $\mathbf{k}_{0}$. The scattered wavefield at $\mathbf{r}$ with wave vector $\mathbf{k}_{r}$ depends on the scattering angle $\theta$, the angle between wave vectors $\mathbf{k}_{0}$ and $\mathbf{k}_{r}$, and on $\rho$, the distance from the scatterer.

of long-period surface waves (periods $\geq 20 \mathrm{~s}$ ) made with large-aperture arrays have shown that the amplitude field is highly oscillating with magnitude of variations that can reach several hundreds percents (e.g., Friederich 1998; Lin et al. 2009; Pollitz 2008; Jin \& Gaherty 2015). However, the estimations of the Laplacian of the amplitude field are usually small compared to the squared gradient of the phase, which often lead to drop the amplitude correction in practice (e.g., Feng \& Ritzwoller 2019). This short overview of the literature suggests that the limits of validity of the eikonal equation are still rather fuzzy. Our motivation is thus to provide new physical insights into the validity of the eikonal equation through a simple theoretical investigation of the problem, which is presented in the sequel.

\section{ERROR DUE TO THE EIKONAL APPROXIMATION}

[Fig. 1]

In this section, we characterize the error that is made by dropping the amplitude term in (5), i.e. by using the eikonal equation instead of the complete Helmholtz equation to derive phase velocities. Following Bodin \& Maupin (2008), we consider the simple case of an incident plane wave that propagates through a medium with background phase velocity $c_{0}$. The wavefield $u_{0}$ at position $\mathbf{r}$ produced by this incident plane wave with wave vector $k_{0}=\omega / c_{0}$ is

$$
u_{0}(\mathbf{r})=\exp \left(i \mathbf{k}_{0} \cdot \mathbf{r}\right)
$$


This wavefield interacts with a scatterer located at $\mathbf{r}_{s}$ and produces the scattered wavefield

$$
u_{s}(\mathbf{r})=\frac{\varepsilon}{\sqrt{2 \pi\left\|\mathbf{r}-\mathbf{r}_{s}\right\|}} \exp \left(i\left[\mathbf{k}_{0} \cdot \mathbf{r}_{s}+\mathbf{k}_{r} .\left(\mathbf{r}-\mathbf{r}_{s}\right)\right]\right)
$$

where $\varepsilon$ is the relative amplitude of the scattered wave field with respect to the incident wavefield, and $\mathbf{k}_{r}$ the wave vector of the scattered wavefield. Figure 1 displays the geometry of this simple ansatz experiment. Assuming that $\varepsilon \ll 1$ and using the Taylor series approximation $(1+x)^{\alpha} \approx 1+\alpha x$, the amplitude of the total wave field is to first order

$$
A=\sqrt{\left\|u_{0}+u_{s}\right\|^{2}} \approx 1+\frac{\varepsilon}{\sqrt{2 \pi \rho}} \cos \left(\mathbf{k}_{s} \cdot \boldsymbol{\rho}\right)
$$

where $\boldsymbol{\rho}=\mathbf{r}-\mathbf{r}_{s}$ and $\mathbf{k}_{s}=\mathbf{k}_{0}-\mathbf{k}_{r}$ is the scattering vector.

We now introduce the new variable $a=\log A$ which, using the Taylor series approximation $\log (1+x) \approx x$, is simply given by

$$
a=\log A \approx \frac{\varepsilon}{\sqrt{2 \pi \rho}} \cos \left(\mathbf{k}_{s} \cdot \boldsymbol{\rho}\right) .
$$

Using $a$, the amplitude correction term in the Helmholtz equation becomes

$$
\frac{\Delta A}{A}=\|\nabla a\|^{2}+\Delta a
$$

The Laplacian of $a$ is

$$
\Delta a \approx \frac{-\varepsilon}{\sqrt{2 \pi \rho}} k_{s}^{2} \cos \left(\mathbf{k}_{s} \cdot \boldsymbol{\rho}\right)
$$

and is thus of order $\varepsilon$. In contrast, because the gradient of $a$ is

$$
\nabla a \approx \frac{-\varepsilon}{\sqrt{2 \pi \rho}} \mathbf{k}_{s} \sin \left(\mathbf{k}_{s} \cdot \boldsymbol{\rho}\right),
$$

the squared gradient is of order $\varepsilon^{2}$ with respect to the incident wave field:

$$
\|\nabla a\|^{2} \approx \frac{\varepsilon^{2}}{2 \pi \rho} k_{s}^{2} \sin ^{2}\left(\mathbf{k}_{s} \cdot \boldsymbol{\rho}\right) .
$$

Its contribution to (11) can thus be neglected to first order. We thus obtain the first-order approximation of the amplitude correction term in the Helmholtz equation:

$$
\frac{\Delta A}{A} \approx \Delta a \approx \frac{-\varepsilon}{\sqrt{2 \pi \rho}} k_{s}^{2} \cos \left(\mathbf{k}_{s} \cdot \boldsymbol{\rho}\right) .
$$

Introducing (15) into the Helmholtz equation (5) gives that

$$
\|\nabla T\|^{-1} \approx c_{0}\left(1+\frac{\Delta a}{k_{0}^{2}}\right)^{-\frac{1}{2}},
$$

which, using the same Taylor series approximation as above, leads to the eikonal phase velocity

$$
c=\|\nabla T\|^{-1} \approx c_{0}\left[1+\frac{1}{2} \frac{\varepsilon}{\sqrt{2 \pi \rho}} \frac{k_{s}^{2}}{k_{0}^{2}} \cos \left(\mathbf{k}_{s} . \boldsymbol{\rho}\right)\right] .
$$


Introducing the angle $\theta$ between $\mathbf{k}_{0}$ and $\mathbf{k}_{r}$ (Fig. 1), we have

$$
k_{s}^{2}=2 k_{0}^{2}(1-\cos \theta)
$$

and

$$
\mathbf{k}_{s} \cdot \boldsymbol{\rho}=k_{0} \rho(\cos \theta-1)
$$

Inserting these terms in (17) we thus finally obtain

$$
\|\nabla T\|^{-1} \approx c_{0}\left[1+\frac{\varepsilon}{\sqrt{2 \pi \rho}}(1-\cos \theta) \cos \left(k_{0} \rho(1-\cos \theta)\right)\right] .
$$

The second term in the right-hand side of (20) approximates the bias that comes from the amplitude term in (5), which results from the contribution of singly scattered waves. It thus approximates the error that is made when the eikonal equation is used to estimate phase velocities.

\section{MITIGATING ERRORS IN EIKONAL TOMOGRAPHY}

Figure 2a shows the apparent eikonal phase velocity model obtained with Eq. (20) considering an incident plane wave with a wavelength of $15 \mathrm{~km}$ (period $5 \mathrm{~s}$ ) and a back azimuth of $270^{\circ}$ propagating in a homogeneous model $\left(c_{0}=3 \mathrm{~km} / \mathrm{s}\right)$ with a scatterer located at the center of the 2-D model. The relative amplitude of the diffracted wave field $\varepsilon$ is $5 \%$. The apparent eikonal velocity model obtained is in excellent agreement with the results of Bodin \& Maupin (2008) (their figure 2). In particular, it reproduces the strong errors resulting from the interference between the incident and back-scattered wave fields, which show oscillations with a spatial periodicity of half the incoming wavelength.

The error in (20) has an oscillatory pattern as a function of the azimuth of the incoming wavefront, which suggests that it can be reduced by azimuthal averaging, as pointed out in Bodin \& Maupin (2008).

The phase velocity map obtained by azimuthal averaging, i.e. integrating (20) over the scattering angle $\theta$, is given by

$$
\frac{1}{2 \pi} \int_{-\pi}^{\pi}\|\nabla T\|^{-1} \mathrm{~d} \theta \approx \frac{1}{2 \pi} \int_{-\pi}^{\pi} c_{0}\left[1+\frac{\varepsilon}{\sqrt{2 \pi \rho}}(1-\cos \theta) \cos \left(k_{0} \rho(1-\cos \theta)\right)\right] \mathrm{d} \theta .
$$

Let us define

$$
I(x)=\frac{1}{2 \pi} \int_{-\pi}^{\pi}(1-\cos \theta) e^{i x(1-\cos \theta)} \mathrm{d} \theta
$$


which, after changing $\theta$ to $\pi-\theta$, can be written

$$
\begin{aligned}
I(x) & =\frac{1}{2 \pi} \int_{0}^{2 \pi}(1+\cos \theta) e^{i x(1+\cos \theta)} \mathrm{d} \theta \\
& =\frac{e^{i x}}{2 \pi}\left[\int_{0}^{2 \pi} e^{i x \cos \theta} \mathrm{d} \theta+\int_{0}^{2 \pi} \cos \theta e^{i x \cos \theta} \mathrm{d} \theta\right] \\
& =e^{i x}\left[J_{0}(x)-i J_{0}^{\prime}(x)\right],
\end{aligned}
$$

where $J_{0}$ is the $0^{t h}$-order Bessel function of the first kind. Inserting the real part of $I\left(k_{0} \rho\right)$ in $(21)$, we obtain

$$
\frac{1}{2 \pi} \int_{0}^{2 \pi}\|\nabla T\|^{-1} \mathrm{~d} \theta=c_{0}\left(1+\frac{\delta c}{c_{0}}\right)
$$

where

$$
\frac{\delta c}{c_{0}} \approx \frac{\varepsilon}{\sqrt{2 \pi \rho}}\left[\cos \left(\rho k_{0}\right) J_{0}\left(\rho k_{0}\right)+\sin \left(\rho k_{0}\right) J_{0}^{\prime}\left(\rho k_{0}\right)\right] .
$$

Equations $(24,25)$ show that after azimuthal averaging, the error on phase velocity oscillates around the true velocity, with a radial pattern centered on the diffracting point (Fig. 2c).

At large distances from the scatterer $(\rho \gg \lambda)$ :

$$
\begin{aligned}
& J_{0}\left(k_{0} \rho\right) \rightarrow \frac{1}{\sqrt{\pi k_{0} \rho}}\left[\cos \left(k_{0} \rho\right)+\sin \left(k_{0} \rho\right)\right] \\
& J_{0}^{\prime}\left(k_{0} \rho\right) \rightarrow \frac{1}{\sqrt{\pi k_{0} \rho}}\left[\cos \left(k_{0} \rho\right)-\sin \left(k_{0} \rho\right)\right]
\end{aligned}
$$

and the correction term in (25) becomes simply

$$
\frac{\delta c}{c_{0}} \approx \frac{\varepsilon}{\sqrt{2 k_{0}} \pi \rho}\left[\sin \left(2 k_{0} \rho\right)+\cos \left(2 k_{0} \rho\right)\right] .
$$

Therefore, the biases in azimuthally averaged eikonal phase velocity maps decrease as a function of $\rho$, the distance from scatterer (Fig. 2c). Moreover, since this error term is dominated by a $\lambda / 2$ periodic pattern, smoothing (or low-pass spatial filtering) is very efficient to attenuate it.

Fig. $2 b$ illustrates the smoothed version of the apparent eikonal velocity model from Fig. 2a, obtained after applying a 2D Gaussian filter of width $\lambda / 2$. Combining lateral and azimuthal averaging of the apparent eikonal phase velocity model strongly attenuates the contribution of the scattered wave field. We obtain a finite-extent blurred anomaly located at the position of the scatterer (Fig. 2d). Note that since these two operations are linear, they can be performed in any order.

[Fig. 2]

\section{NUMERICAL EXPERIMENT}

We now validate numerically our analytical developments by comparing the results of Helmholtz and eikonal tomography in a synthetic experiment. We consider incident plane surface waves at $5 \mathrm{~s}$ period propagating in a model with an average phase velocity of $3.0 \mathrm{~km} / \mathrm{s}$ and velocity perturbations around 

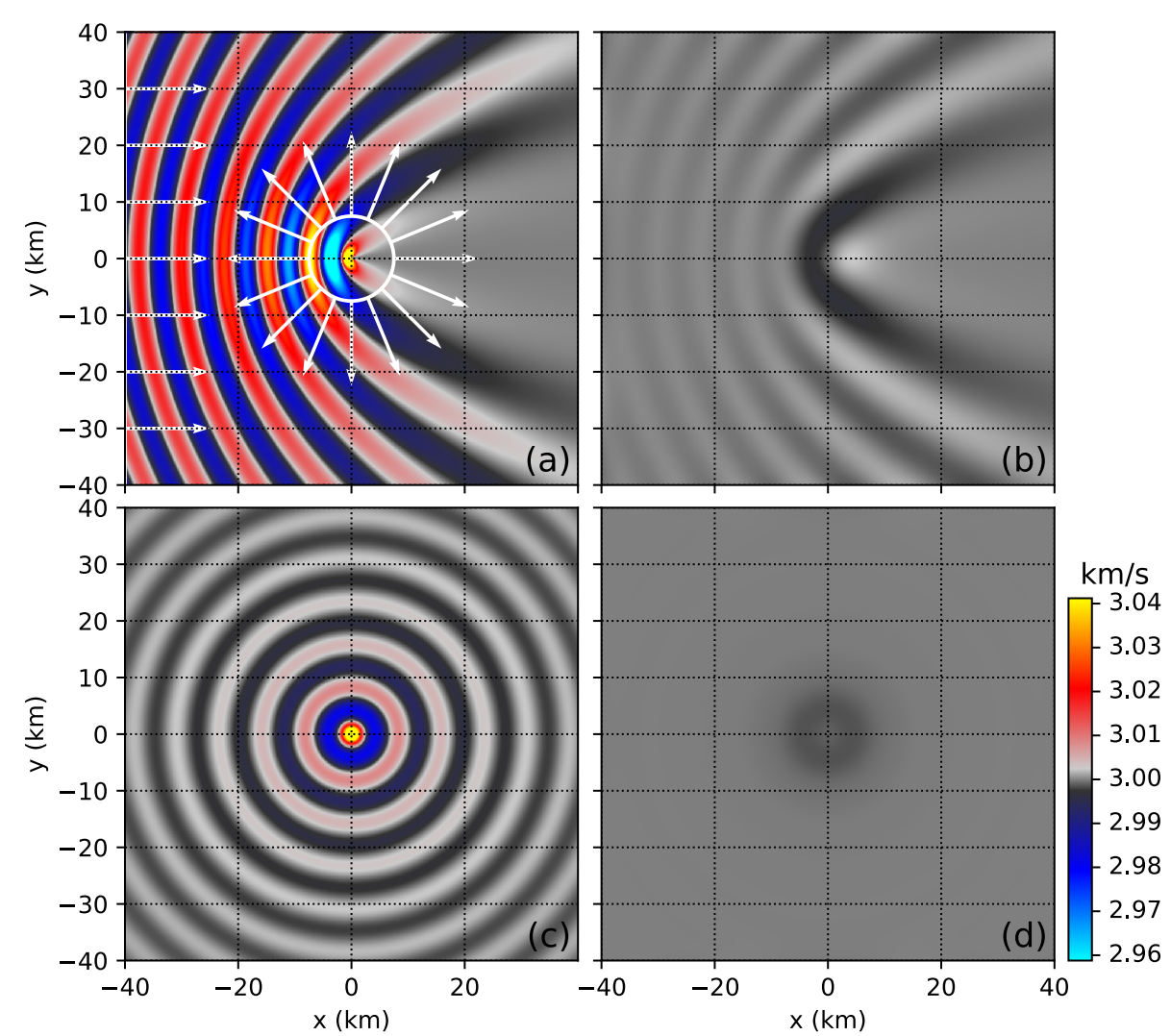

Figure 2. Effects of azimuthal averaging and smoothing on eikonal tomography. (a) Apparent eikonal phase velocity model obtained for a plane wave arriving from back azimuth $270^{\circ}$. The white arrows show the incident and scattered wave fields. The arrow length equates one wavelength. (b) Eikonal model obtained after applying a $\lambda / 2$ Gaussian filter. (c) Azimuthal average of the eikonal models. (d) Azimuthal average of the filtered eikonal models.

$5 \%$ (Fig. 3a). We solve the scalar wave equation with a finite difference method in a regular Cartesian grid. At the boundaries, we impose the displacement produced by an incoming plane wave with a phase velocity of $3.0 \mathrm{~km} / \mathrm{s}$ (wavelength of $15 \mathrm{~km}$ ). We implement Stacey boundary conditions to absorb the outgoing scattered wave field (Stacey 1988). The problem is solved for different azimuths of the incident wave.

Fig. $3 c, d$ show the phase and amplitude fields modeled for an incoming azimuth of $280^{\circ}$ (white arrow). By unwrapping the phase of the complex wave field, we obtain the phase travel times relative to the center of the model (Fig. 3c). The amplitude field is characterized by strong fluctuations (Fig. 3d) caused by focusing/defocusing of the wave front. The location and scale of the amplitude anomalies depend on the azimuth of the incoming plane wave (not shown). Using the two terms of the Helmholtz equation (5) leads to the structural phase velocity model in Fig. 3b, which is very close to the true model (Fig. 3a). 


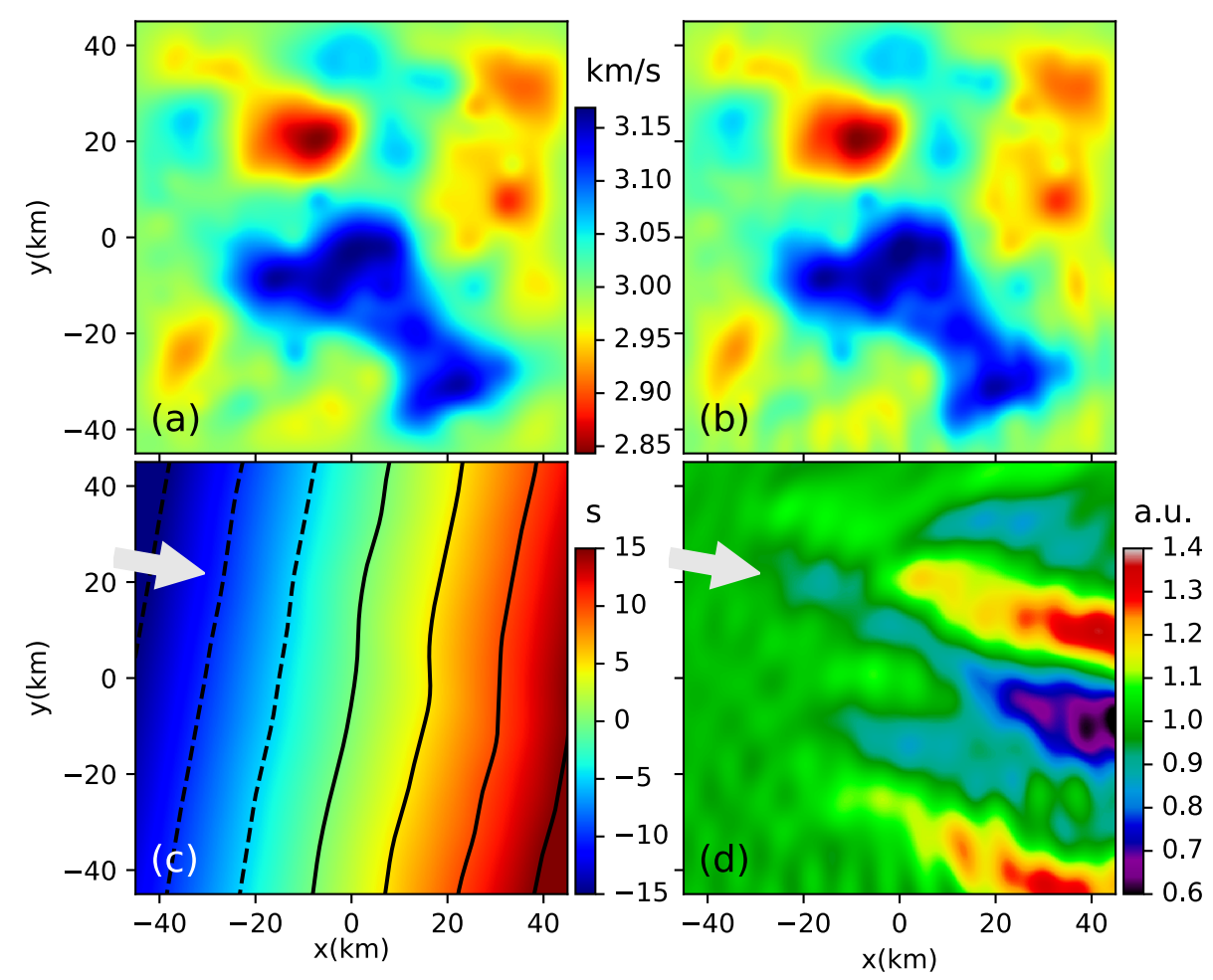

Figure 3. Synthetic numerical Helmholtz tomography experiment. (a) Structural phase velocity model (in km/s). (b) Phase velocity model obtained with the Helmholtz equation using the phase and amplitude fields shown respectively in (c) and (d). (c) Phase travel time of the simulated wave field. Isovalues are indicated with black lines. (d) Amplitude of the simulated wave field in arbitrary units. The white arrows indicate the azimuth of the incident wave $\left(\mathrm{N} 280^{\circ}\right)$.

\section{[Fig. 3]}

Dropping the amplitude correction term of the Helmholtz equation and using only the gradient of the travel time field, we obtain the eikonal phase velocity map shown in Fig. 4a, which differs from the true structural model (Fig. 3a), as emphasized by Wielandt (1993). The biases in the phase velocity map result from the interference between the incident and scattered wave fields. Errors up to $10 \%$ are observed locally (Fig. 4b). Figure 4c shows the eikonal phase velocity model after application of a Gaussian filter which smooths out anomalies smaller than half the propagating wavelength $(7.5 \mathrm{~km})$. Smoothing the phase velocity model reduces the error due to the eikonal approximation, which drops from about $10 \%$ to about $3 \%$ (Figure $4 \mathrm{~d}$ ). The smoothed eikonal model still differs from the expected structural model especially in the regions where the amplitude anomalies are large (for example near the eastern boundary of the model in Fig. 4c).

[Fig. 4]

In Figure 5, we compare the structural phase velocity model (Fig. 5a), the eikonal model obtained from a single azimuth $\left(280^{\circ}\right.$, Fig. 5 b), the eikonal model obtained after averaging all azimuths with 


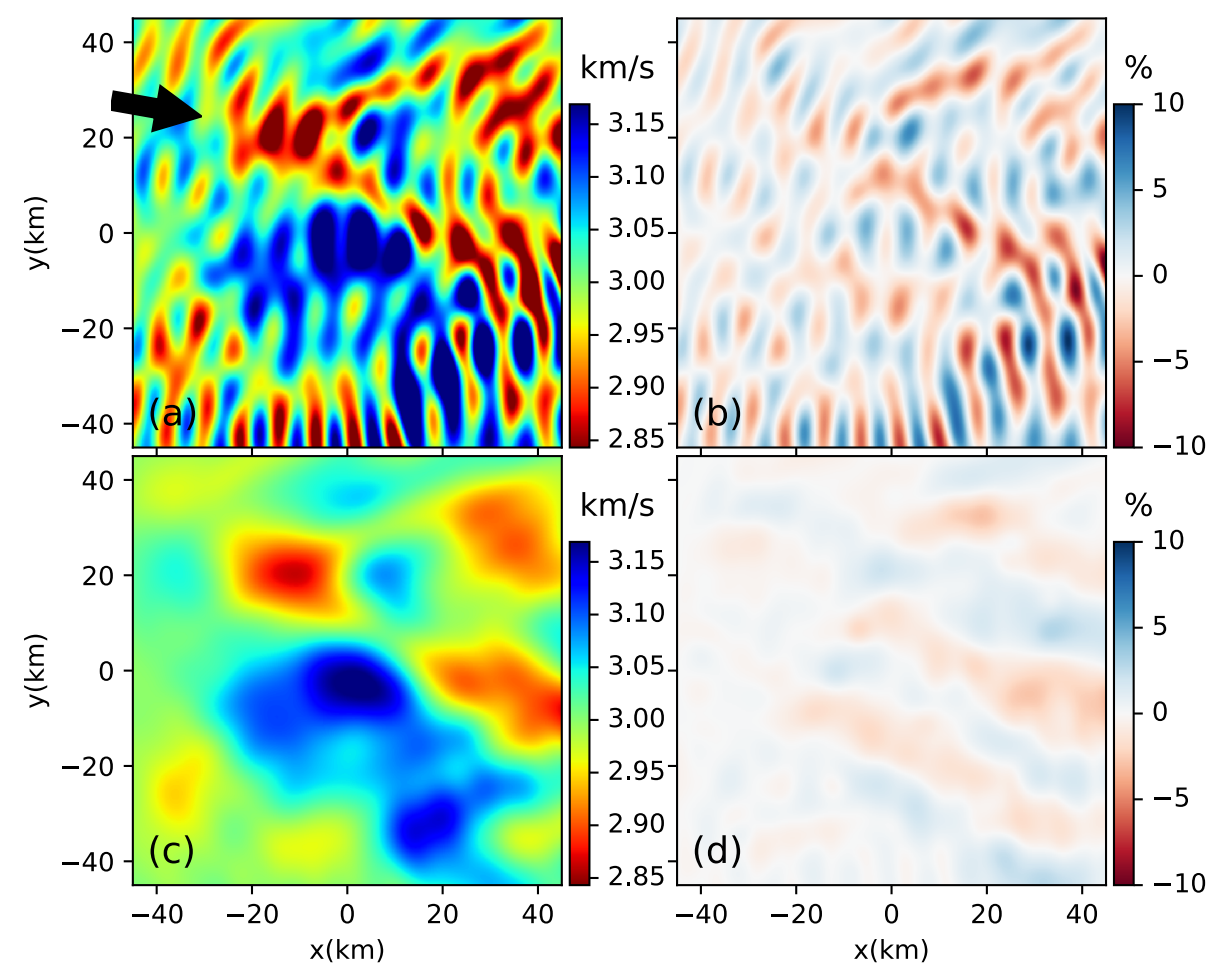

Figure 4. (a) Eikonal phase velocity model obtained using only the gradient of the phase field shown in Fig. 3c. (b) Error of the eikonal phase velocity model shown in (a), expressed in percents. (c) Eikonal phase velocity after applying a $\lambda / 2$ Gaussian filter. (d) Error of the filtered eikonal model.

an increment of $2.5^{\circ}$ (Fig. 5c), and the model obtained after both azimuthal averaging and Gaussian filtering (Fig. 5d). This test confirms the benefit of azimuthal averaging to minimize the errors due to the eikonal approximation, which drop to less than $2 \%$ after azimuthal averaging (Fig. 5c) and $1.1 \%$ after Gaussian smoothing (Fig. 5d).

[Fig. 5]

\section{CONCLUSIONS}

Our results reconcile the sometimes contradictory results that can be found in the literature regarding the reliability of eikonal tomography. Indeed, neglecting the amplitude term in the Helmholtz equation leads to velocity models with strong artefacts (Wielandt 1993). However, when measurements obtained on waves coming from different azimuths are combined, which is typically the case in most tomographic studies, the errors are dramatically reduced. Applying a low-pass Gaussian filter can further reduce the errors in phase velocity models. This additional spatial filtering does not degrade the resolution since the cut-off can be set at half the dominant propagating wavelength. The resolution 


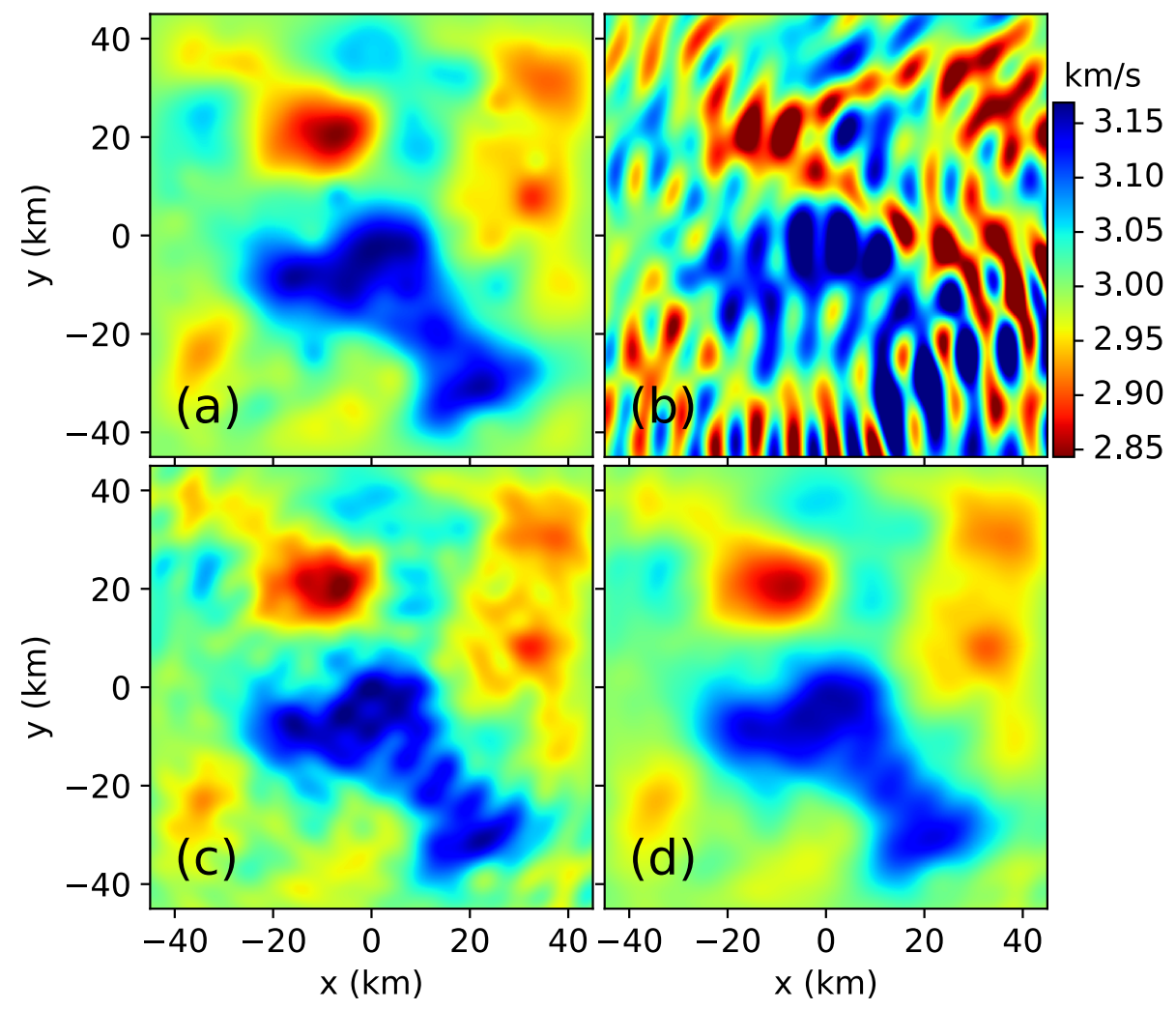

Figure 5. (a) Structural phase velocity model (in $\mathrm{km} / \mathrm{s}$ ). (b) Eikonal tomography model obtained using a single incoming plane wave from back azimuth $280^{\circ}$ (c) Phase velocity model obtained after azimuthal averaging of the eikonal tomographic models. (d) Eikonal model obtained after azimuthal averaging and Gaussian filtering.

potential of eikonal tomography thus remains close to the theoretical resolution limit of full waveform inversion (Virieux \& Operto 2009).

\section{ACKNOWLEDGMENTS}

We thank Emmanuel Kaestle and Malcolm White for their thoughtful and constructive reviews. This work is part of the OROGEN research project, a tripartite partnership between the CNRS, TOTAL and BRGM.

\section{REFERENCES}

Bodin, T. \& Maupin, V., 2008. Resolution potential of surface wave phase velocity measurements at small arrays, Geophys. J. Int., 172, 698-706.

Feng, L. \& Ritzwoller, M. H., 2019. A 3-D shear velocity model of the crust and uppermost mantle beneath Alaska including apparent radial anisotropy, J. Geophys. Res., 124, 10468-10497. 
Friederich, W., 1998. Wave-theoretical inversion of teleseismic surface waves in a regional network: phasevelocity maps and a three-dimensional upper-mantle shear-wave-velocity model for southern Germany, Geophys. J. Int., 132, 203-225.

Friederich, W. \& Dalkolmo, J., 1995. Complete synthetic seismograms for a spherically symmetrical Earth by a numerical computation of the Green's function in the frequency-domain, Geophys. J. Int., 122, 537-550.

Friederich, W. \& Wielandt, E., 1995. Interpretation of seismic surface waves in regional networks: joint estimation of wavefield geometry and local phase velocity. Methhod and numerical tests, Geophys. J. Int., 120, 731-744.

Friederich, W., Wielandt, E., \& Stange, S., 1994. Non-plane geometry of seismic surface wavefields and their implications for regional surface-wave tomography, Geophys. J. Int., 119, 931-948.

Friederich, W., Hunzinger, S., \& Wielandt, E., 2000. A note on the interpretation of seismic surface waves over three-dimensional structures, Geophys. J. Int., 143, 335-339.

Jin, G. \& Gaherty, J. B., 2015. Surface wave phase-velocity tomography based on multichannel crosscorrelation, Geophys. J. Int., 201, 1383-1398.

Lin, F. C. \& Ritzwoller, M. H., 2011. Helmholtz surface wave tomography for isotropic and azimuthally anisotropic structure, Geophys. J. Int., 186, 1104-1120.

Lin, F. C., Ritzwoller, M. H., \& Snieder, R., 2009. Eikonal tomography: Surface wave tomography by phase front tracking across a regional broad-band seismic array, Geophys. J. Int., 177, 1091-1110.

Lin, F. C., Tsai, V. C., \& Ritzwoller, M. H., 2012. The local amplification of surface waves: A new observable to constrain elastic velocities, density, and anelastic attenuation, J. Geophys. Res., 117, B06302, doi:10.1029/2012JB009208.

Pollitz, F. F., 2008. Observations and interpretation of fundamental mode Rayleigh wavefields recorded by the Transportable Array (USArray), J. Geophys. Res., 113, doi:10.1029/2007JB005556.

Pollitz, F. F. \& Snoke, J. A., 2010. Rayleigh-wave phase velocity maps and three-dimensional shear velocity structure of the western US from local non-plane surface wave geometry, Geophys. J. Int., 180, 1153-1169.

Roy, I. G., 2015. On computing first and second order derivative spectra, J. Comp. Phys., 295, 307-321.

Scales, J. A. \& Tenorio, L., 2001. Prior information and uncertainty in inverse problems, Geophysics, 66, 389-397.

Shen, W. \& Ritzwoller, M. H., 2016. Crustal and uppermost mantle structure beneath the United States, J. Geophys. Res., 121, doi:10.1002/2016JB012887.

Stacey, R., 1988. Improved transparent boundary formulations for the elastic wave equation, Bull. Seismol. Soc. Am., 78, 2089-2097.

Tanimoto, T., 1990. Modelling curved surface wave paths: membrane surface wave synthetics, Geophys. J. Int., 102, 89-100.

Tromp, J. \& Dahlen, F. A., 1993. Variational principles for surface wave propagation on a laterally heterogeneous Earth - III. Potential representation, Geophys. J. Int., 112, 195-209.

Červený, V., 2001. Seismic Ray Theory, Cambridge University Press. 
Virieux, J. \& Operto, S., 2009. An overview of full-waveform inversion in exploration geophysics, Geophysics, 74, WCC1-WCC26.

Wielandt, E., 1993. Propagation and structural interpretation of non-plane waves, Geophys. J. Int., 113, 45-53.

This paper has been produced using the Blackwell Scientific Publications GJI LTEX2e class file. 